\title{
Experimenting Data Integration with DIS@DIS
}

\author{
Andrea Calì, Domenico Lembo, Riccardo Rosati, and Marco Ruzzi \\ Dipartimento di Informatica e Sistemistica \\ Università di Roma "La Sapienza" \\ Via Salaria 113, I-00198 Roma, Italy \\ \{cali, lembo, rosati,ruzzi\}adis.uniroma1.it
}

\begin{abstract}
Data integration consists in providing a uniform access to a set of heterogeneous sources through a common representation called global schema. In this paper we present DIS@DIS, a data integration system that adopts innovative techniques for query answering in a complex integration environment. In particular, DIS@DIS is able to deal with integrity constraints, which are used to enhance the expressiveness of the global schema. Since data at the sources may not satisfy the constraints, DIS@DIS is capable of reasoning in the presence of incomplete and inconsistent information, so as to provide consistent answers to user queries. Moreover, DIS@DIS is able to deal with both local-as-view and global-as-view approaches for the specification of the mapping between the global schema and the sources. DIS @DIS incorporates novel optimization techniques for query processing, which speed up query answering time even in the presence of complex global schemata and large amounts of data. Indeed, we show experimental results that prove the feasibility of our approach.
\end{abstract}

\section{Introduction}

The recent development of Information Technology has provided the availability of a huge amount of information, stored in sources that are often autonomous, heterogeneous, and both physically and logically distributed. Data integration consists in providing a uniform access to different sources; it has emerged as an important issue in the areas of distributed databases, cooperative information systems, data warehousing, and Web data management.

In data integration $[17,11,15]$ the user is offered a common representation of the underlying data, called global schema; the user sees and queries the global schema as a single database schema, while the data integration system carries out the task of suitably querying the underlying sources and assembling the results in order to answer user queries. Integrity constraints are expressed on the global schema to enhance its expressiveness, yet the data at the sources may not satisfy such constraints.

Another crucial aspect of query processing in data integration is the specification of the relationship between the global schema and the sources, called mapping. Two basic approaches are possible for the mapping: the global-as-view (GAV), in which elements of the global schema are associated to views over the sources, and the local-as-view $(L A V)$ which requires the sources to be associated to views over the global schema [15].

A. Persson and J. Stirna (Eds.): CAiSE 2004, LNCS 3084, pp. 51-66, 2004.

(c) Springer-Verlag Berlin Heidelberg 2004 
In this paper we present DIS@DIS ${ }^{1}$, a system for semantic data integration, that is capable of reasoning about integrity constraints in order to improve query answering. A significant issue here is that in DIS@DIS it is assumed that the sources are incomplete, i.e., they do not provide all information needed to answer user queries. In such a setting, due to the presence of integrity constraints, query answering amounts to reasoning about inconsistent and incomplete information, which is a difficult task. There are several data integration systems in the literature [7, 18, 12,16,13,10,2,4]; yet, to the best of our knowledge, only IBIS [4] is able to reason about integrity constraints, though in a limited setting w.r.t. DIS@DIS. We emphasize that query processing in DIS@DIS is mostly carried out at the intensional (i.e., schema) level; in particular, DIS@DIS takes integrity constraints into account by reformulating each user query into a new one in which information about integrity constraints is encoded. Keeping most of query processing at the intensional level improves efficiency, since the size of the schema and constraints is usually much smaller than the size of the data.

The main innovative and distinguishing features of DIS@DIS are the following:

1. DIS @DIS is able to reason about a very expressive class of constraints in a relational setting, namely key dependencies (KDs), inclusion dependencies (IDs), which are a generalization of foreign key dependencies, and exclusion dependencies (EDs), which establish disjointness between projections on relations.

2. DIS@DIS deals with incompleteness of data (violations of IDs) by reformulating user queries according to the IDs; the technique adopted, based on [6], takes advantage of novel optimization techniques.

3. DIS@DIS is capable of dealing with inconsistencies of data (violations of KDs and EDs) on the basis of a novel semantics [6], namely the loosely-sound semantics, that allows to obtain consistent answers from inconsistent data. DIS@DIS implements novel optimization techniques for checking the consistency of data, which is a costly step in query processing.

4. DIS@DIS is able to deal with both GAV and LAV mapping specifications. In particular, it implements a novel ad-hoc technique for LAV mappings, and, when only IDs and EDs are expressed over the global schema, it is able to transform a LAV system into a GAV one that is equivalent to the original w.r.t. query answering, thus allowing for the use of the techniques developed for GAV. The transformation technique extends that presented in [3], which does not directly allow for query processing and is limited to a restricted form of mapping views. Moreover, the technique of [3] works only for LAV integration systems without constraints.

In order to show the effectiveness and efficiency of the techniques implemented in DIS@DIS, we have tested the system in a real-world setting; the experimental results have shown that in practical cases DIS@DIS is able to answer queries in reasonable time, thus proving the feasibility of our approach.

The rest of the paper is organized as follows. In Section 2 we present the formal framework for data integration adopted in DIS@DIS; in Section 3 we present the query answering techniques of the system; in Section 4 we present the architecture of DIS @DIS;

\footnotetext{
${ }^{1}$ The name of the system is an acronym for Data Integration System at the Dipartimento di Informatica e Sistemistica.
} 
in Section 5 we present the result of experiments that have been we have carried out on a real-world case. Section 6 concludes the paper.

\section{Framework for Data Integration}

In this section we present the framework for data integration adopted in DIS@DIS; such a framework is based on the relational model with integrity constraints. we assume that the reader is familiar with the basic notions of relational databases [1].

Syntax. A relational database schema $\mathcal{R}$ is a set of relation symbols, each with an associated arity, which is an integer value greater or equal than 1 that denotes the number of its attributes; to denote that a relation symbol $R$ has arity $n$, we will write it as $R / n$. Formally, a data integration system $\mathcal{I}$ is a triple $\langle\mathcal{G}, \mathcal{S}, \mathcal{M}\rangle$, where:

1. $\mathcal{G}$ is the global schema expressed in the relational model with integrity constraints. Different integrity constraints are expressed over $\mathcal{G}$; in particular we have:

(i) A set of inclusion dependencies $\Sigma_{I}$, i.e., a set of assertions of the form $R_{1}[\mathbf{A}] \subseteq$ $R_{2}[\mathbf{B}]$, where $R_{1}, R_{2}$ are relations in $\mathcal{G}, \mathbf{A}=A_{1}, \ldots, A_{n}(n \geq 0)$ is a sequence of attributes of $R_{1}$, and $\mathbf{B}=B_{1}, \ldots, B_{n}$ is a sequence of distinct attributes of $R_{2}$. Note that we allow repetition of attributes in the left-hand side of the inclusion $^{2}$.

(ii) A set of key dependencies $\Sigma_{K}$, i.e., a set of assertions the form $k e y(R)=\mathbf{A}$, where $R$ is a relation in $\mathcal{G}$, and $\mathbf{A}=A_{1}, \ldots, A_{n}$ is a sequence of attributes of $R$. We assume that at most one $\mathrm{KD}$ is specified for each relation.

(iii) A set of exclusion dependencies $\Sigma_{E}$, i.e., a set of assertions of the form $R_{1}[\mathbf{A}] \cap$ $R_{2}[\mathbf{B}]=\emptyset$, where $R_{1}$ and $R_{2}$ are relations in $\mathcal{G}, \mathbf{A}=A_{1}, \ldots, A_{n}$ and $\mathbf{B}=$ $B_{1}, \ldots, B_{n}$ are sequences of attributes of $R_{1}$ and $R_{2}$, respectively.

2. $\mathcal{S}$ is the source schema, constituted by the schemata of the different sources. We assume that the sources are relational; considering only relational sources is not a restriction, since we may assume that sources that are not relational are suitably presented in relational form by wrappers.

3. $\mathcal{M}$ is the mapping between $\mathcal{G}$ and $\mathcal{S}$, specifying the relationship between the global schema and the source schema. Our framework allows for the specification of two kind of mapping: the global-as-view $(G A V)$, in which global relations are defined in terms of the sources, and the local-as-view ( $L A V)$, in which, conversely, source relations are defined in terms of the global schema. We assume that views in the mapping are expressed in the language of conjunctive queries (CQ) [1] for LAV, and union of conjunctive queries (UCQ) for GAV. A UCQ of arity $n$ is a set of conjunctive queries $Q$ such that each $q \in Q$ has the same arity $n$ and uses the same predicate symbol in the head. More specifically:

(i) A GAV mapping is a set of assertions of the form $\left\langle R_{\mathcal{G}}, \varphi_{\mathcal{S}}\right\rangle$, where $R_{\mathcal{G}} / n$ is a relation of $\mathcal{G}$ and $\varphi_{\mathcal{S}}$ is a UCQ of arity $n$ over $\mathcal{S}$.

(ii) A LAV mapping is a set of assertions of the form $\left\langle R_{\mathcal{S}}, \varphi_{\mathcal{G}}\right\rangle$, where $R_{\mathcal{S}} / n$ is a relation of $\mathcal{S}$ and $\varphi_{\mathcal{G}}$ is a CQ of arity $n$ over $\mathcal{G}$.

${ }^{2}$ For details about such dependencies, that have the same properties as the standard ones, we refer the reader to [1]. 
Now we come to queries expressed over the global schema; in our setting, we assume that such queries are expressed in the language of union of conjunctive queries.

Semantics. A database instance (or simply database) $\mathcal{C}$ for a relational schema $\mathcal{R}$ is a set of facts of the form $R(t)$ where $R$ is a relation of arity $n$ in $\mathcal{R}$ and $t$ is an $n$-tuple of constants. We denote as $R^{\mathcal{C}}$ the set of tuples of the form $\{t \mid R(t) \in \mathcal{C}\}$, and with $Q^{\mathcal{C}}$ the result of the evaluation of the query $Q$ (expressed over $\mathcal{R}$ ) on $\mathcal{C}$.

Now we come to the semantics of a data integration system $\mathcal{I}=\langle\mathcal{G}, \mathcal{S}, \mathcal{M}\rangle$. Such a semantics is defined by first considering a source database for $\mathcal{I}$, i.e., a database $\mathcal{D}$ for the source schema $\mathcal{S}$. We call global database for $\mathcal{I}$ any database for $\mathcal{G}$. Given a source database $\mathcal{D}$ for $\mathcal{I}=\langle\mathcal{G}, \mathcal{S}, \mathcal{M}\rangle$, the semantics $\operatorname{sem}(\mathcal{I}, \mathcal{D})$ of $\mathcal{I}$ w.r.t. $\mathcal{D}$ is the set of global databases for $\mathcal{I}$ such that:

1. $\mathcal{B}$ satisfies the integrity constraints $\Sigma_{I}, \Sigma_{K}$ and $\Sigma_{E}$ in $\mathcal{G}$; we refer the reader to [1] for the notion of satisfaction of IDs, KDs and EDs.

2. $\mathcal{B}$ satisfies $\mathcal{M}$ w.r.t. $\mathcal{D}$. In particular,

(i) $\mathcal{B}$ satisfies a GAV assertion $\left\langle R_{\mathcal{G}}, \varphi_{\mathcal{S}}\right\rangle$ w.r.t. $\mathcal{D}$ if $\left(\varphi_{\mathcal{S}}\right)^{\mathcal{D}} \subseteq\left(R_{\mathcal{G}}\right)^{\mathcal{B}}$;

(ii) $\mathcal{B}$ satisfies a LAV assertion $\left\langle R_{\mathcal{S}}, \varphi_{\mathcal{G}}\right\rangle$ w.r.t. $\mathcal{D}$ if $\left(R_{\mathcal{S}}\right)^{\mathcal{D}} \subseteq\left(\varphi_{\mathcal{G}}\right)^{\mathcal{B}}$.

Note that the above definition amounts to consider the mapping as sound but not necessarily complete; intuitively, for each mapping formula, the data retrievable at the sources are a subset of the data that satisfy the corresponding fragment of global schema. Such an assumption allows us to suitably model data incompleteness.

We now give the semantics of queries posed to a data integration system. Formally, given a source database $\mathcal{D}$ for $\mathcal{I}$ we call certain answers to a query $q$ of arity $n$ w.r.t. $\mathcal{I}$ and $\mathcal{D}$, the set $\operatorname{cert}(q, \mathcal{I}, \mathcal{D})=\left\{\bar{t} \mid\right.$ for each $\left.\mathcal{B} \in \operatorname{sem}(\mathcal{I}, \mathcal{D}), \bar{t} \in q^{\mathcal{B}}\right\}$.

\section{Query Answering in DIS@DIS}

In this section we present the techniques for query answering adopted in the DIS@DIS system.

The presence of integrity constraints on the global schema complicates the task of query answering; the key issue here is that in our framework it is possible that the semantics $\operatorname{sem}(\mathcal{I}, \mathcal{D})$ of a data integration system $\mathcal{I}$ w.r.t. a source database $\mathcal{D}$ is constituted by an infinite set of databases that may have infinite size [15].

\subsection{Query Answering in GAV}

In order to deal with inclusion dependencies, DIS@DIS makes use of the algorithm IDrewrite [6], which is able to rewrite the user query according to the IDs expressed over the global schema. When processing a user query in the presence of IDs alone, DIS@DIS first executes IDrewrite, and then processes the obtained query as if there were no IDs; the properties of the algorithm IDrewrite ensure that the answers obtained in this way are the certain answers to the query. The execution of IDrewrite, which basically encodes the information about the IDs into the reformulated query, overcomes the violations of IDs, which are a form of incompleteness of the data w.r.t. the IDs. 
Example 1. Consider a data integration system $\mathcal{I}=\langle\mathcal{G}, \mathcal{S}, \mathcal{M}\rangle$ with the following global schema $\mathcal{G}$ :

$$
\begin{aligned}
& \text { player(Pname, Pteam }) \\
& \text { team(Tname, Tcity })
\end{aligned}
$$

The following ID is defined over $\mathcal{G}$ :

$$
\text { player }[\text { Pteam }] \subseteq \text { team }[\text { Tname }]
$$

Consider a query $q(X) \leftarrow \operatorname{team}(X, Y)$ issued by the user over $\mathcal{G}$ and asking for all team names. The rewritten query is $q^{\prime}(X) \leftarrow \operatorname{team}(X, Y) \vee \operatorname{player}(W, X)$. Intuitively, in case of violations of the ID, instead of operating on the data, $q^{\prime}$ seeks for team names in relation team and also in the second column of player; in fact, if there are violations of the ID, there are team names appearing in player and not appearing in team.

In the presence of KDs together with IDs, the possible presence of violations, which are a form of inconsistency of the data w.r.t. the KDs, forces us to verify the satisfaction of KDs by the data at the sources. First of all, we have to restrict our attention to a special class of constraints, since the problem of query answering in the presence of general KDs and IDs is undecidable [5,6]. In particular, we consider the class of non-key-conflicting IDs (NKCIDs), which is a class of IDs that generalizes foreign key dependencies, together with KDs: in this case query answering is decidable [5]. Here the notion of retrieved global database $(R G D B)$ comes into play; given a data integration system $\mathcal{I}=\langle\mathcal{G}, \mathcal{S}, \mathcal{M}\rangle$ and a source database $\mathcal{D}$, the retrieved global database $\operatorname{ret}(\mathcal{I}, \mathcal{D})$ is the minimal global database w.r.t. set containment that satisfies the mapping $\mathcal{M}$ w.r.t. $\mathcal{D}$; it is obtained by evaluating, for each global relation in $\mathcal{G}$, the corresponding mapping view over $\mathcal{D}$. If the RGDB satisfies $\Sigma_{K}$, we can proceed as in the absence of KDs; on the contrary, a single violation of a KD leads, in the sound semantics, to a trivial case (since $\operatorname{sem}(\mathcal{I}, \mathcal{D})=\emptyset$, any tuple of the proper arity is in the answer to the query); the same holds if we add EDs on the global schema, together with NKCIDs and KDs. This property makes the sound semantics not suitable in practical cases, where we adopt the loosely-sound semantics [6]. In the loosely-sound semantics, the certain answers to a query are those that are in the answer to the query over all global databases which are consistent with the integrity constraints and that are obtained with a minimal repair of the violations of KDs and EDs.

Example 2. Recall Example 1, with the additional constraint

$$
\text { key }(\text { player })=\{\text { Pname }\}
$$

The source schema is $\left\{s_{1} / 3, s_{2} / 2, s_{3} / 2\right\}: s_{1}$ stores name, team and age of players; $s_{2}$ stores name and city of teams, and $s_{3}$ stores name and team of players. The mapping associates to player the UCQ

$$
\text { player }(X, Y) \leftarrow s_{1}(X, Y, Z) \vee s_{3}(X, Y)
$$

and to team the query

$$
\operatorname{team}(X, Y) \leftarrow s_{2}(X, Y)
$$


Consider a source database $\mathcal{D}$ with $\left(s_{1}\right)^{\mathcal{D}}=\{\langle$ figo, realMadrid, 31$\rangle\},\left(s_{2}\right)^{\mathcal{D}}=$ $\{\langle$ realMadrid, Madrid $\rangle\}, s_{3}=\{\langle$ totti, roma $\rangle,\langle$ figo, cavese $\rangle\}$. The retrieved global database is as follows:

\begin{tabular}{c|c|c|}
\hline \multirow{4}{*}{ player } & figo & realMadrid \\
\hline totti & roma \\
\hline figo & cavese \\
\hline
\end{tabular}

team realMadrid $\mid$ madrid

As for the violation of the KD, there are two ways of repairing the RGDB in a minimal way: either we eliminate the fact player(figo, realMadrid) or the fact player(figo,cavese). Violations of the ID are instead repaired by adding facts to the RGDB. We have two forms of global databases in $\operatorname{sem}(\mathcal{I}, \mathcal{D})$ :

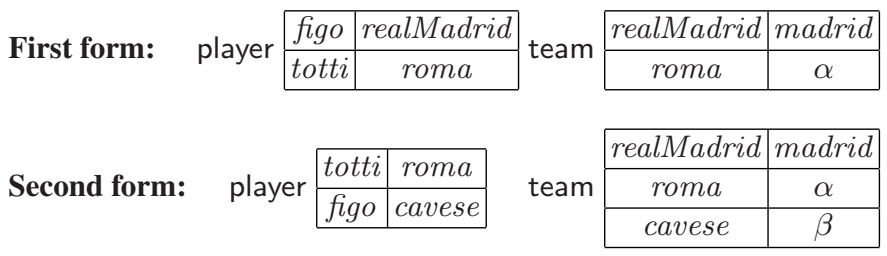

where $\alpha, \beta$ are constants. Considering the query $Q(X) \leftarrow$ team $(X, Y)$, we have that the certain answers to $Q$ under the loosely-sound semantics are $\{\langle$ roma $\rangle,\langle$ realMadrid $\rangle\}$.

We point out that DIS@DIS does not actually compute all the repairs of the RGDB, but resorts to an intensional rewriting approach: DIS @DIS first checks whether $\operatorname{ret}(\mathcal{I}, \mathcal{D})$ satisfies the sets of KDs and EDs on $\mathcal{G}^{3}$; if this happens, it proceeds with the reformulation with IDrewrite and with the evaluation over the RGDB. If not, the user query is reformulated in a different query, expressed in Datalog with negation under stable model semantics $[14,8]$, a well-known extension of Datalog. The reformulated query is then evaluated over the RGDB by means of the deductive database system DLV [9].

When only IDs are expressed on the global schema, the consistency check is not necessary. In this case, after IDs are taken into account with the execution of IDrewrite, we can simply unfold the reformulated query; unfolding, which is the traditional technique for GAV systems without integrity constraints [15], amounts to substitute each atom in the query with the associated mapping view. Finally, the unfolded query can be evaluated over the source database.

\subsection{Query Answering in LAV}

Now we come to the case where the data integration system has a LAV mapping.

When the consistency check is necessary, i.e., when KDs and EDs are expressed on the global schema, the RGDB can be calculated in LAV systems as well. If the RGDB satisfies KDs and EDs, we can proceed as in the GAV case. Otherwise, we are able to do query answering in the case of violations of KDs and EDs, but this feature is not yet implemented in DIS@DIS.

${ }^{3}$ Notice that this step requires the computation of the closure of KDs and EDs w.r.t. logical implication. 
When only IDs are expressed over the global schema, instead of computing the RGDB, which is a costly operation, DIS@DIS transforms the LAV system into a GAV one which is equivalent w.r.t. query answering [3]. Note that the transformation is done "at compile time", i.e. it does not depend on the queries, but it involves only the specification of the system. The transformation introduces an additional set of IDs on the global schema, which however can be treated together with the pre-existing ones; therefore, we are able to apply the same techniques as in the GAV case.

\subsection{Optimizations in GAV}

The experiments carried out with DIS @DIS have shown that the most costly step from a computational point of view is the generation of the RGDB (see Section 5). In the presence of a GAV mapping specification, optimizations are possible, that can actually speed up the generation of the RGDB. We have focused our attention on the GAV approach, which is used by the majority of data integration system, for example MOMIS [2] and IBIS [4].

The key point in the optimization is the loosely-sound semantics, which allows us to consider only a limited portion of the RGDB in the consistency check. In particular, we need to take into account only the tuples that can contribute to the answer and those that may conflict with such tuples. In the following, we will denote as RDGB the portion of it that is relevant to a certain query. First of all, given a user query $Q$, we can disregard global relations which do not appear in $Q$. For the remaining global relations, instead of retrieving the whole set of tuples for each one of them, we "push-down" the selections of $Q$, thus reducing the number of retrieved tuples. At this point, for each queried source $R$, if the previous selection was not performed on the key of $R$ or on a subset of it, we need to retrieve the tuples that conflict with the ones already retrieved. This is done by selecting the tuples of $R$ that share the same key with the ones already retrieved.

Example 3. Consider a data integration system $\mathcal{I}=\langle\mathcal{G}, \mathcal{S}, \mathcal{M}\rangle$, with $\mathcal{G}=$ $\left\{R_{1} / 1, R_{2} / 2, R_{3} / 2\right\}, \operatorname{key}\left(R_{2}\right)=\{1\}$ and $\mathcal{S}=\left\{S_{1} / 2, S_{2} / 1, S_{3} / 2\right\}$. The mapping $\mathcal{M}$ associates to $R_{1}$ the query $R_{1}(X) \leftarrow S_{1}(X, Y), S_{2}(Y)$, to $R_{2}$ the query $R_{2}(X, Y) \leftarrow S_{3}(X, Y)$, and to $R_{3}$ the query $R_{3}(X, Y) \leftarrow S_{1}(Y, X)$. Suppose we have the user query $Q(X) \leftarrow R_{1}(X), R_{2}(X, a)$.

To compute the retrieved global database, we can ignore $R_{3}$, since it does not appear in the body of $Q$. As for $R_{1}$, we have to populate it entirely, without optimizations, since there is no selection over it. For $R_{2}$ we have a selection on a non-key attribute; we first populate it with the tuples obtained by evaluating the following query over the source database: $Q_{R_{2}}\left(X_{1}, X_{2}\right) \leftarrow S_{3}\left(X_{1}, a\right)$, where we have pushed down the selection on $R_{2}$ in $Q$. After that, we still have to retrieve the tuples that are in conflict with the ones retrieved by $Q_{R_{2}}$; this is done by evaluating the query $Q_{R_{2}}^{\prime}$ over the source database, where $Q_{R_{2}}^{\prime}$ is as follows: $Q_{R_{2}}^{\prime}\left(X_{1}, X_{2}\right) \leftarrow S_{3}\left(X_{1}, a\right), S_{3}\left(X_{1}, X_{2}\right)$. At this point we can proceed in the evaluation of the certain answers to $Q$ as described in Section 3.1. The extension of $R_{2}$ is the union of the answers to $Q_{R_{2}}$ and $Q_{R_{2}}^{\prime}$.

\section{System Architecture}

In this section we present the architecture of DIS @ DIS. Figure 1 shows an overall picture of the system and of its modules. 


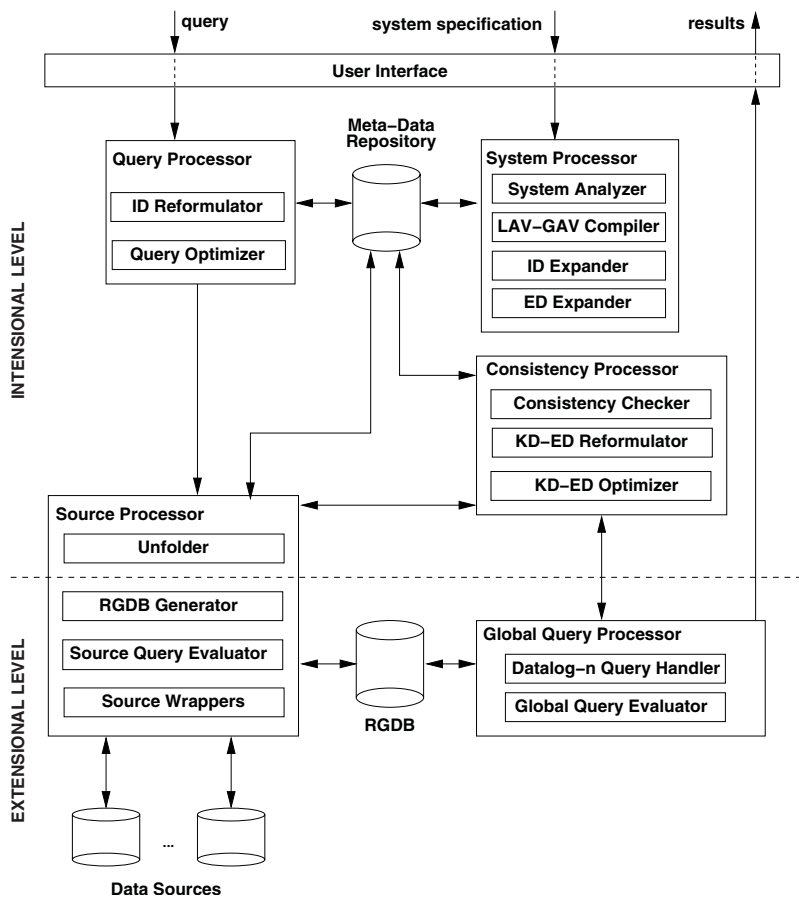

Fig. 1. Architecture of the system

In the system, the Meta-Data Repository stores an internal representation of the intensional information, i.e., the system specification and the user queries. Also, a User Interface takes care of the interaction with the system user.

The system architecture comprises five main modules:

1. the System Processor elaborates and reasons about the specification of the data integration system;

2. the Query Processor has the task of elaborating the user queries, according to IDs expressed on the global schema;

3. the Source Processor unfolds the query when only IDs are defined over the global schema. On the contrary, when the consistency check is necessary, it executes the reformulated query, by retrieving at the sources the data relevant for the query, and by storing such data in the RGDB. In this module, source wrappers provide a relational view of the sources (source schema $\mathcal{S}$ );

4. the Consistency Processor is activated only when KDs or EDs are defined on the global schema. It detects the situations in which data stored in the RGDB are not consistent with the key dependencies and the exclusion dependencies and, if needed, computes a further reformulation of the query.

5. the Global Query Processor evaluates the reformulated query over the RGDB.

In this section we briefly describe the main components of the system. 


\subsection{System Processor}

System Analyzer. This module verifies the properties of the data integration system. In particular: (i) it verifies whether the system is non-key-conflicting; (ii) based on the constraints and the mapping, it establishes whether two global relations are equivalent or contained one into the other.

LAV-GAV Compiler. The task of the LAV-GAV Compiler is to transform a specification of a LAV system into a specification of GAV system which is equivalent to the initial one w.r.t. query processing. We recall that the transformation is performed in a purely intensional fashion, and only once for any integration system specification (unless the specification changes).

ID Expander. In order to speed up the query reformulation phase, DIS@DISprocesses the IDs by computing the closure of them w.r.t. implication [1]. The computational cost of this calculation is, in the worst case, exponential in the size of the initial set of IDs; however, since this operation is to be performed only once, i.e. when the specification of a system is loaded, such an exponential blow-up is of not critical from a practical point of view. In order to further optimize the computation of the closure, indexing structures are used: their introduction allows us to avoid useless application on the implication rule. The process terminates when no new IDs can be added.

ED Expander. The ED Expander has the task of inferring new exclusion dependencies starting from the given set of EDs and IDs. Such dependencies are obtained through a recursive inference procedure that computes the closure of the initial set of EDs w.r.t. implication [1]. Such a process is necessary to efficiently deal with inconsistent data, since it reduces the computational cost of the operations performed by the Consistency Processor.

\subsection{Query Processor}

ID Reformulator. This module implements the algorithm IDrewrite [6], by producing a rewriting of any query posed to the system by the user.

The algorithm implemented by the ID Reformulator is actually an optimization of the procedure ID-rewrite presented in [6], which exploits the fact that the inclusion set has been previously closed by the ID Expander.

Query Optimizer. The Query Optimizer performs an optimization of the rewritten query obtained by the ID Reformulator. In particular, it can check a priori whether a query will not produce any result; in this case the query is immediately excluded. This module is currently under development.

\subsection{Source Processor}

Unfolder. This module unfolds the query produced by the ID Reformulator according to the mapping assertions, i.e., reformulates the query in terms of the source relations.

RGDB Generator. The RGDB Generator builds the RGDB from the system specification and the source data. For GAV systems, the optimized technique described in Section 3 is used. For LAV systems, the generator builds the RGDB by extracting data from the sources, and suitably adding tuples in the global relations for each extracted tuple. 
Source Wrappers. Such modules provide a relational interface to data sources, which in general may be semi-structured and non-relational.Wrappers for DIS @ DIS are currently under development.

\subsection{Consistency Processor}

Consistency Checker. The Consistency Checker analyzes the RGDB to detect the presence of violations of key and exclusion dependencies expressed over the global schema. In such a case, the KD-ED Reformulator is invoked.

KD-ED Reformulator. This module reformulates the query in order to deal with violations of KDs and EDs, under the loosely-sound semantics (see Section 3), and produces a program expressed in Datalog with negation.

KD-ED Optimizer. This module carries out the task of optimizing the rewriting produced by the KD-ED Reformulator. Indeed, it restricts the rewritten query by eliminating those parts of the query that cannot actually contribute to the answer, by checking a priori whether a subquery will not produce any result. This module is currently under development.

\subsection{Global Query Processor}

The task of this module is to evaluate the rewritten query generated by the Query Processor and the Consistency Processor over the RGDB. If the rewriting is a Datalog query with negation, then the evaluation is performed by the Datalog-n-Query Handler, that makes use of the Disjunctive Datalog engine DLV [9], otherwise the query is a standard union of conjunctive queries, therefore the rewriting can be evaluated by the Global Query Evaluator, that translates the query in SQL and then passes this query to the DBMS (currently, MySQL) that stores the RGDB.

\section{Experimental Results}

We have carried out experiments on the DIS @ DIS system in order to test the feasibility of our approach. We have built a GAV data integration system using a set of data belonging to the information system of the University of Rome "La Sapienza"; such data contain information about students, professors and exams between 1993 and 1998. The source data come from different databases and are significantly overlapping. The source schema comprises 27 relations, while the global schema has 10 relations. Key dependencies and inclusion dependencies are expressed on the global schema; the set of dependencies is quite complex: in particular, the IDs are cyclic, and the semantics of the system includes global databases of infinite size.

We have deployed the DIS@DIS system, which is written in Java, on an Intel Pentium III machine, with $800 \mathrm{MHz}$ processor clock frequency, equipped with $640 \mathrm{Mb}$ of RAM, and with a $40 \mathrm{~Gb}$ hard disk drive at $7200 \mathrm{RPM}$; the machine runs the operating system Windows XP. In order to test the efficiency of our system independently of network delays, the data sources have been migrated to a local DBMS, thus avoiding delays due to remote sources. Our DBMS of choice is MySQL, which we chose for its efficiency and simplicity. 
The global schema of the system we built for our experiments comprises the following relations:

student(ID, FirstName, SecondName, CityOfResidence, Address, Telephone,

HighSchoolSpecialization)

enrollment (StudentID, FacultyName, Year)

exam(Code, Description)

professor(FirstName, SecondName, Area)

university (Code, City, Name)

master_exam_record(StudentID, Exam, ProfFirstName, ProfSecondName, Mark,

Date, CourseYear)

bachelor_exam_record(StudentID, ExamCode, Session, Mode, Mark)

teaching(Exam, ProfFirstName, ProfSecondName, Academic Year)

exam_plan(Code, StudentID, PlanType, RequestDate, Status)

exam_plan_data(Code, ExamName, Exam Type)

We have the following constraints (KDs and IDs) over the global schema:

$$
\begin{array}{ll}
\operatorname{key}(\text { student })=\{1\} & \operatorname{key}(\text { exam })=\{1\} \\
\operatorname{key}(\text { professor })=\{1,2\} & \operatorname{key}(\text { university })=\{1\}
\end{array}
$$

master_exam_record $[1] \subseteq$ student $[1]$

master_exam_record $[2] \subseteq$ exam[1]

teaching $[1] \subseteq$ master_exam_record $[2]$

master_exam_record $[3,4] \subseteq$ professor $[1,2]$

professor $[1,2] \subseteq$ teaching $[2,3]$

$$
\begin{array}{r}
\text { bachelor_exam_record }[1] \subseteq \text { student }[1] \\
\text { bachelor_exam_record }[2] \subseteq \text { exam }[1] \\
\text { exam_plan }[2] \subseteq \text { student }[1] \\
\text { exam_plan_data[1] } \subseteq \text { exam_plan }[1]
\end{array}
$$

We have tested the system with 7 queries, which we describe below. Note that, for the sake of conciseness, we do not specify projections on the result for those queries where this choice has no consequences on the performances. The queries below are written in SQL.

$\left(Q_{1}\right)$ This query returns the exams in the exam plan of the student with ID 09089903. SELECT *

FROM exam_plan_data D, exam_plan P WHERE D. code = P. code AND P.studentID = '09089903';

$\left(Q_{2}\right)$ This query returns the exams passed by the student with ID 09089903.

\section{SELECT *}

FROM master_exam_record $V$, exam $E$ WHERE V.exam = E. code AND V.studentID = '09089903';

$\left(Q_{3}\right)$ Similar to the previous one, but returns also personal data of the student.

\section{SELECT *}

FROM master_exam_record $V$, exam $E$, student $S$ WHERE V.exam = E. code AND V.studentID $=$ S.ID and $S \cdot I D=' 09089903^{\prime}$; 
$\left(Q_{4}\right)$ This query returns the information about students and their exam plans, for students who have as first name Federico and who have chosen the plan type T1.

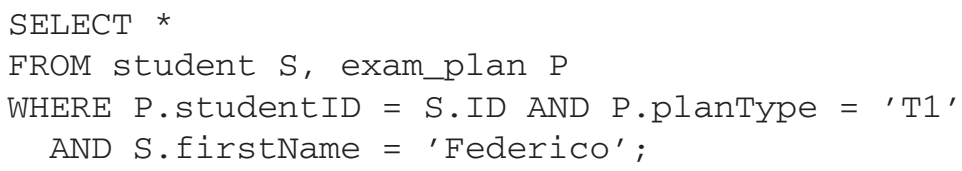

$\left(Q_{5}\right)$ This query returns the second name of professors that teach a course and whose first name is Mario. The query has been chosen to test both the algorithm IDrewrite and the optimization technique described in Section 3.3.

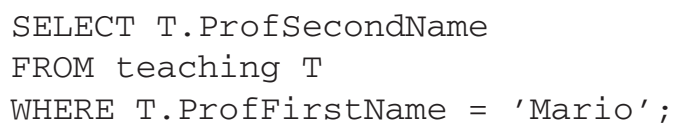

$\left(Q_{6}\right)$ This query returns the information about students with second name Rossi and the master exams they have passed.

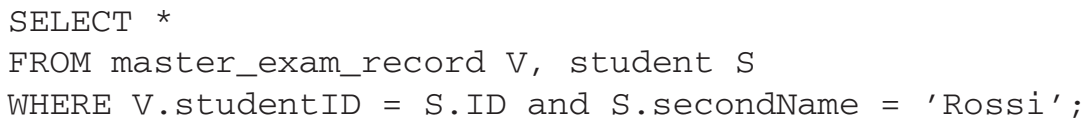

$\left(Q_{7}\right)$ This query retrieves the information about students living in Roma and their exam plans; it was chosen to test the behaviour of the system when the number of tuples in the result is high.

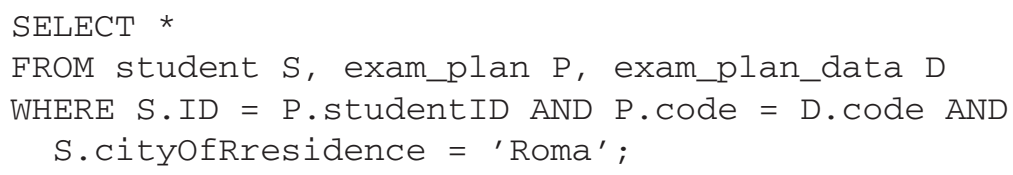

Now we come to the results we have obtained with the above queries. The optimization technique allows us to significantly reduce the number of tuples of the RGDB retrieved by the RGDB Generator, and thus the time needed for the retrieval. As a consequence, also the consistency check time is reduced, as well as the query evaluation time.

The comparison has been done between the basic optimization technique, that consists in considering only the global relations that appear in the global query, and the full optimization technique, both described in Section 3.3. The experimental data are summarized in Figure 2. The values obtained with the basic optimization are denoted as "old" in the figure.

Note that the bottleneck in query answering is the generation of the RGDB, which requires the processing of a significant quantity of data. The preprocessing of the query and its evaluation requires a time which is negligible w.r.t. the operation of retrieving the RGDB and checking the satisfaction of the constraints. The query evaluation time (in milliseconds) and the number of retrieved tuples for the different queries is reported ${ }^{4}$ in

\footnotetext{
${ }^{4}$ We do not have bar charts here because the values would not be all readable on the same scale.
} 

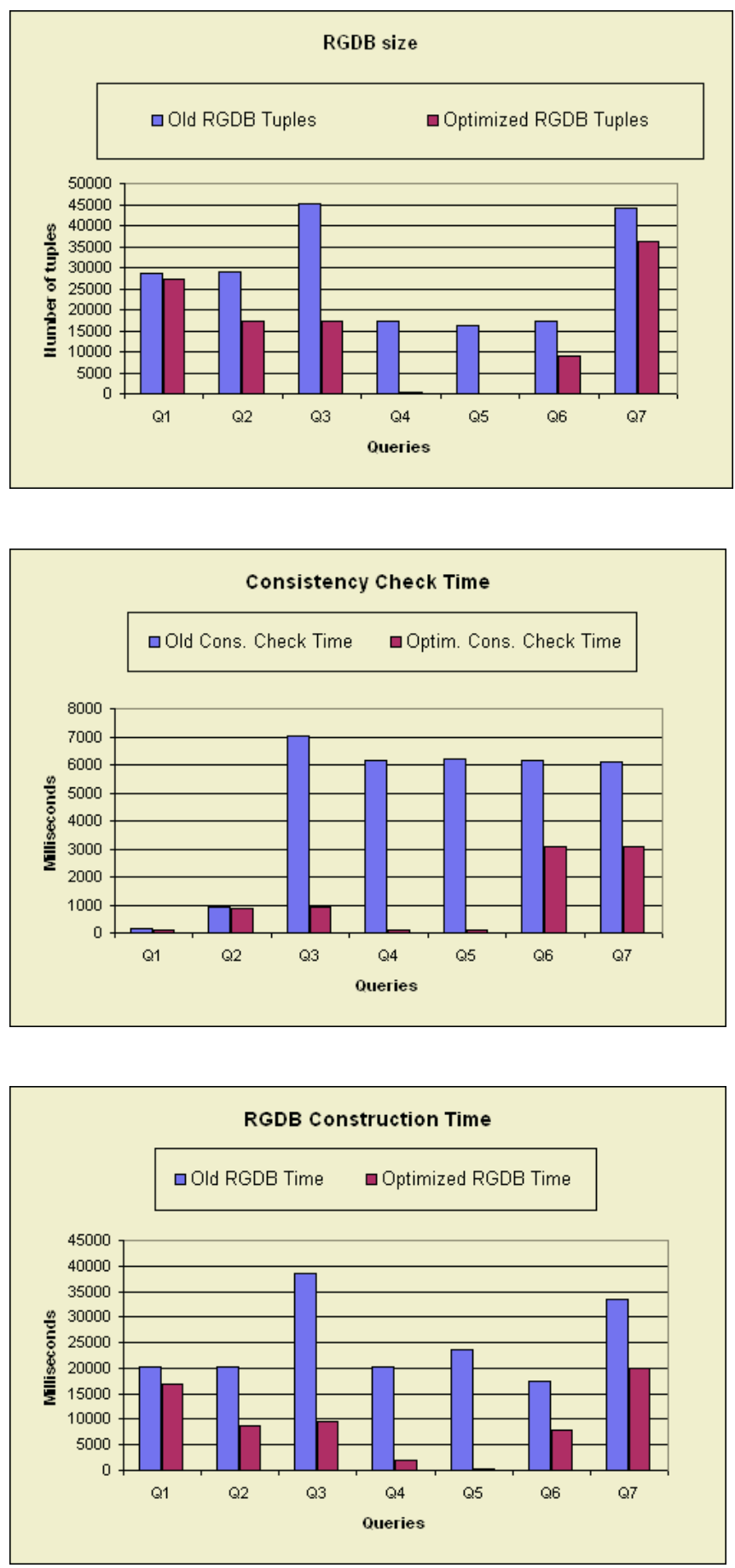

Fig. 2. Experimental results for query answering in DIS@DIS 


\begin{tabular}{|l|ccccccc|}
\hline & $Q_{1}$ & $Q_{2}$ & $Q_{3}$ & $Q_{4}$ & $Q_{5}$ & $Q_{6}$ & $Q_{7}$ \\
\hline Exec. time without optim. & 181 & 30 & 70 & 20 & 10 & 1030 & 17345 \\
Exec. time with optim. & 70 & 20 & 41 & 10 & 8 & 746 & 16834 \\
\hline
\end{tabular}

\begin{tabular}{|l|lllllll|}
\hline & $Q_{1}$ & $Q_{2}$ & $Q_{3}$ & $Q_{4}$ & $Q_{5}$ & $Q_{6}$ & $Q_{7}$ \\
\hline No. of tuples in the result & 19 & 7 & 7 & 1 & 13 & 212 & 5119 \\
\hline
\end{tabular}

Fig. 3. Execution time (in milliseconds) and no. of retrieved tuples for the queries considered in the experiments

Figure 3. The time needed for overall query processing, including generation of RGDB and consistency check, was acceptable in all cases.

Before concluding, we point out that there are 13 tuples in the answer to the query $Q_{5}$. This result would not have been achieved by disregarding IDs and simply evaluating the query with classical unfolding, which actually retrieves only 5 tuples. In this case, the algorithm IDrewrite rewrites $Q_{5}$ in the union of conjunctive queries reported below, whose evaluation over the RGDB produces the 15 tuples that are the certain answers to he query.

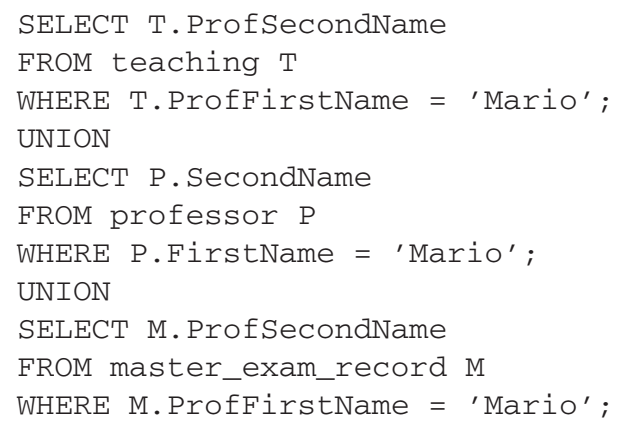

\section{Conclusions}

In this paper we have presented DIS@DIS, a system for semantic data integration that implements state-of-the-art techniques for query processing under integrity constraints. To the best of our knowledge, DIS@DIS is the first system that enables query processing under constraints in both LAV and GAV specifications and that is able to deal with inconsistent data. In partiular, DIS@DIS reasons about integrity constraints expressed on the global schema, so as to provide consistent answers in the presence of incomplete and inconsistent data.

A significant issue is that DIS@DIS keeps the computation as much as possible at the intensional level. In this way, the use of computations involving data is reduced, thus improving efficiency. In fact, the size of the data is usually much larger than the size of the intensional specification of the system, and moreover source accesses typically represent a bottleneck in data integration systems. As for computations involving data, DIS@DIS exploits novel optimization techniques. 
We have tested DIS@DIS on a real-world case, showing that the system is able to perform well on a large amount of data, in the presence of redundancies and inconsistenies, and under a complex set of constraints over the global schema. Response times to queries has proved to be acceptable in all tests we have carried out.

\section{Acknowledgments}

This research has been supported by the Projects INFOMIX (IST-2001-33570) and SEWASIE (IST-2001-34825) funded by the EU, and by the Project MAIS funded by MIUR (Ministero per l'Istruzione, l'Università e la Ricerca).

\section{References}

1. Serge Abiteboul, Richard Hull, and Victor Vianu. Foundations of Databases. Addison Wesley Publ. Co., 1995.

2. Sonia Bergamaschi, Silvana Castano, Maurizio Vincini, and Domenico Beneventano. Semantic integration of heterogeneous information sources. Data and Knowledge Engineering, 36(3):215-249, 2001.

3. Andrea Calì, Diego Calvanese, Giuseppe De Giacomo, and Maurizio Lenzerini. On the expressive power of data integration systems. In Proc. of ER 2002, 2002.

4. Andrea Calì, Diego Calvanese, Giuseppe De Giacomo, Maurizio Lenzerini, Paolo Naggar, and Fabio Vernacotola. IBIS: Semantic data integration at work. In Proc. of CAiSE 2003, pages 79-94, 2003.

5. Andrea Calì, Domenico Lembo, and Riccardo Rosati. On the decidability and complexity of query answering over inconsistent and incomplete databases. In Proc. of PODS 2003, pages 260-271, 2003.

6. Andrea Calì, Domenico Lembo, and Riccardo Rosati. Query rewriting and answering under constraints in data integration systems. In Proc. of IJCAI 2003, 2003.

7. Sudarshan S. Chawathe, Hector Garcia-Molina, Joachim Hammer, Kelly Ireland, Yannis Papakonstantinou, Jeffrey D. Ullman, and Jennifer Widom. The TSIMMIS project: Integration of heterogeneous information sources. In Proc. of the 10th Meeting of the Information Processing Society of Japan (IPSJ'94), pages 7-18, 1994.

8. Thomas Eiter, Georg Gottlob, and Heikki Mannilla. Disjunctive Datalog. ACM Trans. on Database Systems, 22(3):364-418, 1997.

9. Thomas Eiter, Nicola Leone, Cristinel Mateis, Gerald Pfeifer, and Francesco Scarcello. The KR system dlv: Progress report, comparison and benchmarks. In Proc. of KR'98, pages 636-647, 1998.

10. Cheng Hian Goh, Stéphane Bressan, Stuart E. Madnick, and Michael D. Siegel. Context interchange: New features and formalisms for the intelligent integration of information. ACM Trans. on Information Systems, 17(3):270-293, 1999.

11. Alon Y. Halevy. Answering queries using views: A survey. VLDB Journal, 10(4):270-294, 2001.

12. Joachim Hammer, Hector Garcia-Molina, Jennifer Widom, Wilburt Labio, and Yue Zhuge. The Stanford data warehousing project. IEEE Bull. on Data Engineering, 18(2):41-48, 1995.

13. Matthias Jarke, Maurizio Lenzerini, Yannis Vassiliou, and Panos Vassiliadis, editors. Fundamentals of Data Warehouses. Springer, 1999.

14. Phokion G. Kolaitis and Christos H. Papadimitriou. Why not negation by fixpoint? J. of Computer and System Sciences, 43(1):125-144, 1991. 
15. Maurizio Lenzerini. Data integration: A theoretical perspective. In Proc. of PODS 2002, pages 233-246, 2002.

16. Yannis Papakonstantinou, Hector Garcia-Molina, and Jennifer Widom. Object exchange across heterogeneous information sources. In Proc. of ICDE'95, pages 251-260, 1995.

17. Jeffrey D. Ullman. Information integration using logical views. In Proc. of ICDT'97, volume 1186 of $L N C S$, pages 19-40. Springer, 1997.

18. Jennifer Widom (ed.). Special issue on materialized views and data warehousing. IEEE Bull. on Data Engineering, 18(2), 1995. 